\title{
Correction to: A class of topological groups which do not admit normal compatible locally quasi-convex topologies
}

\section{Elena Martín-Peinador ${ }^{1}$ D Víctor Pérez Valdés $^{2}$}

Published online: 23 June 2018

(c) Springer-Verlag Italia S.r.I., part of Springer Nature 2018

\section{Correction to: RACSAM} https://doi.org/10.1007/s13398-018-0507-y

Unfortunately an erratum appears in the statement corresponding to Theorem 6.9 of the above mentioned paper.

It is written there: $|J| \leq \mathfrak{c}$.

It should read: $|J|=\mathfrak{c}$. After this modification, the proof stated for 6.9 is correct.

The original article can be found online at https://doi.org/10.1007/s13398-018-0507-y.

$凶$ Elena Martín-Peinador

peinador@ucm.es

Víctor Pérez Valdés

vperez03@ucm.es

1 Instituto de Matemática Interdisciplinar y Departamento de Álgebra, Geometría y Topología, Facultad de Matemáticas, Universidad Complutense de Madrid, 28040 Madrid, Spain

2 Departamento de Álgebra, Geometría y Topología, Facultad de Matemáticas, Universidad Complutense de Madrid, 28040 Madrid, Spain 